Article

\title{
Nonlinear Modeling and Coordinate Optimization of a Semi-Active Energy Regenerative Suspension with an Electro-Hydraulic Actuator
}

\author{
Farong Kou *, Jiafeng Du, Zhe Wang, Dong Li and Jianan Xu \\ College of Mechanical Engineering, Xi'an University of Science and Technology, Xi'an 710054, China; \\ 18710407191@163.com (J.D.); wzwczl@163.com (Z.W.); 18700788272@163.com (D.L.); alang41@163.com (J.X.) \\ * Correspondence: koufarong@xust.edu.cn
}

Received: 17 December 2017; Accepted: 15 January 2018; Published: 23 January 2018

\begin{abstract}
In order to coordinate the damping performance and energy regenerative performance of energy regenerative suspension, this paper proposes a structure of a vehicle semi-active energy regenerative suspension with an electro-hydraulic actuator (EHA). In light of the proposed concept, a specific energy regenerative scheme is designed and a mechanical properties test is carried out. Based on the test results, the parameter identification for the system model is conducted using a recursive least squares algorithm. On the basis of the system principle, the nonlinear model of the semi-active energy regenerative suspension with an EHA is built. Meanwhile, linear-quadratic-Gaussian control strategy of the system is designed. Then, the influence of the main parameters of the EHA on the damping performance and energy regenerative performance of the suspension is analyzed. Finally, the main parameters of the EHA are optimized via the genetic algorithm. The test results show that when a sinusoidal is input at the frequency of $2 \mathrm{~Hz}$ and the amplitude of $30 \mathrm{~mm}$, the spring mass acceleration root meam square value of the optimized EHA semi-active energy regenerative suspension is reduced by $22.23 \%$ and the energy regenerative power RMS value is increased by $40.51 \%$, which means that while meeting the requirements of vehicle ride comfort and driving safety, the energy regenerative performance is improved significantly.
\end{abstract}

Keywords: semi-active suspension; feed energy; parameter optimization; genetic algorithm

\section{Introduction}

A suspension system is a key component of a vehicle's chassis system, and its performance directly determines the ride comfort, operational stability, and driving safety of the vehicle. The performance of traditional passive suspension without adjustable parameters is insufficient to meet the higher requirements $[1,2]$. With the application of sensor technology and control technology, controllable suspension with superior performance has attracted more and more attention. Although the traditional controllable suspension can realize real-time adjustment of the suspension's performance, it is limited by its high cost and high energy consumption [3-5].

The type of energy regenerative active suspension raised provides a method to solve the above mentioned problems, that is, through the regeneration of a vehicle's vertical vibration energy caused by an uneven road surface, the heat energy dissipated by the suspension's shock absorber in the form of friction is transformed into a recyclable power through the energy recovery device, which is used for active suspension active control and utilization, thereby reducing the problem of the large energy consumption of the active suspension [6-8]. At present, the research on energy regenerative suspension systems is mainly focused on how to improve the efficiency of energy regeneration, while ignoring the original design of the suspension and lacking a coordination analysis of the suspension system's damping performance and energy regenerative performance [9-11]. 
Zuo et al. $[12,13]$ designed a type of gear rack energy regenerative suspension, carried out theoretical and experimental studies, and analyzed the relationship between vehicle ride comfort and handling stability and the suspension's energy regenerative performance. The bench test results show that the suspension is effective for vibration attenuation and the vibration energy regenerative efficiency is improved. Fan et al. [14] integrated a ball screw and a direct current (DC) motor into a suspension, optimized the damping characteristics of the suspension, and recovered the vibration energy. Yu et al. [15] put forward a gear rack energy regenerative suspension, and set up a clutch mechanism in the motor and the gear rack mechanism. The simulation analysis results show that it can regenerate energy at the same time, basically meeting the requirements of vehicle ride comfort and handling stability.

On the basis of the above research, this paper proposes a structure of a vehicle semi-active energy regenerative suspension with an electro-hydraulic actuator (EHA) in Section 2. A quarter-car model with two degrees of freedom is built in Section 3.1; then, a mechanical properties test is carried out, and according to the test results, we perform the parameter identification for the system model using a recursive least squares algorithm in Section 3.2; and the nonlinear model of the semi-active energy regenerative suspension with an EHA is established in Section 3.3. In Section 4, a LQG control strategy for the semi-active energy regenerative suspension with an EHA is designed. Moreover, the influences of the main parameters of the EHA on the damping performance and energy regenerative performance of the suspension are analyzed in Section 5.1; then, the main parameters of the EHA are optimized via the genetic algorithm in Section 5.2; and the bench test is carried out in Section 5.4. Finally, conclusions are summarized in Section 6.

\section{Structure and Principle of the Semi-Active Energy Regenerative Suspension with an EHA}

The basic structure of the semi-active energy regenerative suspension system with an EHA is shown in Figure 1. The system is mainly composed of a spiral spring, a hydraulic cylinder, a hydraulic motor, a brushless DC motor, a digital signal processor controller, a composite energy recovery device, and a digital potentiometer and the corresponding sensor. The hydraulic cylinder uses a double pole double-acting symmetrical hydraulic cylinder; the hydraulic motor uses a gear motor that can carry out positive inversion; and the brushless DC generator uses a permanent magnet brushless DC generator [16].

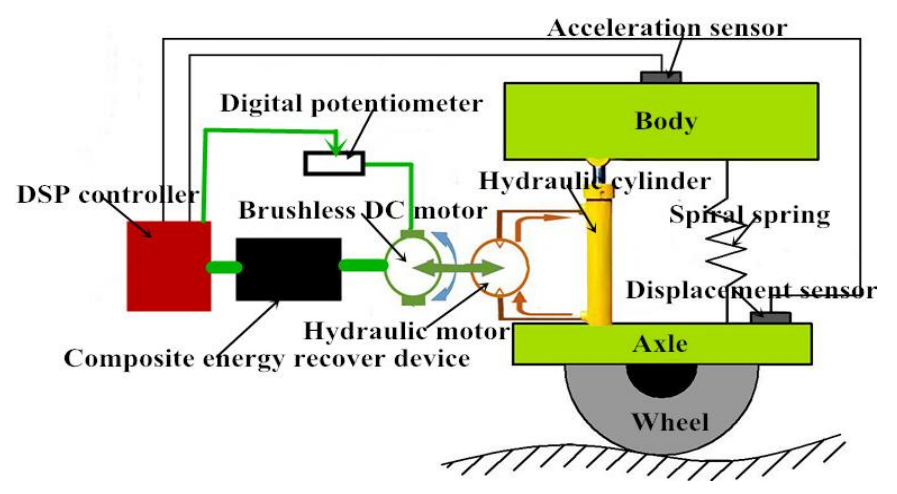

Figure 1. The structure of the semi-active energy regenerative suspension with an electro-hydraulic actuator (EHA). DC: direct current.

When the vehicle is running, the hydraulic cylinder moves with the body vibration and pushes hydraulic oil to drive the hydraulic motor to rotate, the output shaft of the hydraulic motor drives the coaxial brushless DC generator by coupling, and the generated electrical energy is recovered and stored by a composite energy recovery device to realize energy regeneration in the EHA energy regenerative suspension. At the same time, the sensor sends a signal of the vehicle's operation condition 
to the controller. According to the control strategy, the controller changes the external load resistance value of the brushless DC motor by adjusting the value of the digital potentiometer. Therefore, the electromagnetic torque of the motor can be changed, and the damping force of the hydraulic cylinder can be controlled to realize the control function of the EHA semi-active suspension.

The composite energy recovery device is shown in Figure 2. The device is mainly composed of a three-phase rectifier, a filter circuit, boost module 1, boost module 2, MOSFET A, MOSFET B, a voltage equalizing circuit, a super capacitor group, a voltage sensor, a DSP controller, a voltage stabilizing circuit, a diode, and a storage battery. Among them, the super capacitor group consists of four capacitors from the $2.7 \mathrm{~V} 100 \mathrm{~F}$ super capacitor series, in which the voltage is $10.8 \mathrm{~V}$, the capacity is $25 \mathrm{~F}$, and the optimal charging voltage is $9-10 \mathrm{~V}$. Boost module 1 boosts to $9 \mathrm{~V}$ for super capacitor charging. Referring to the vehicle battery, the lead-acid battery used is $12 \mathrm{~V} 8$ ampere-hour, in which the optimal charging voltage is $14.4 \pm 0.2 \mathrm{~V}$. Booster module 2 boosts to $14.4 \mathrm{~V}$ for charging the battery after the voltage stabilizing circuit. In order to prevent the battery from over-charging the super capacitor, a unidirectional conducting diode is connected in series between the voltage stabilizing circuit and the storage battery.

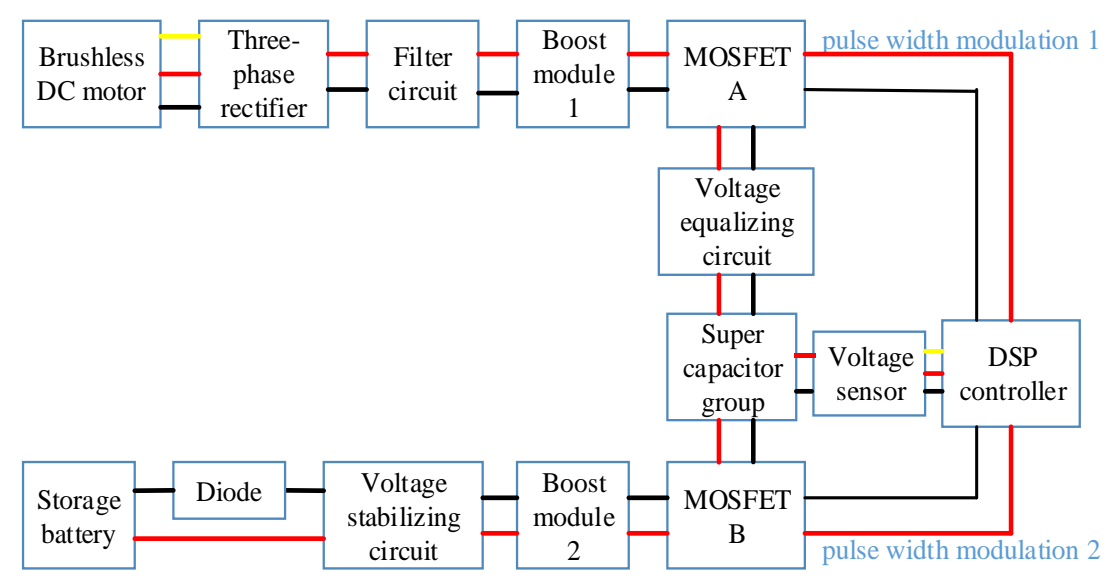

Figure 2. Illustrative diagram of the complex energy recovery device.

The specific working process of the composite energy recovery device is as follows. The voltage sensor connected in parallel at both ends of the super capacitor group is detected in real-time by the super capacitor group's voltage signal, which is collected in the DSP controller by the A/D sampling module of the DSP controller. When the voltage at both ends of the super capacitor group is detected to reach $4 \mathrm{~V}$, the DSP controller outputs two pulse width modulation signals. One output, pulse width modulation 1, is high and it drives the MOSFET switch A to open. The other output, pulse width modulation 2, is low and it drives the MOSFET switch B to close. At this moment, the super capacitor group is in charge status and the three-phase alternating current generated by the brushless DC motor through a three-phase rectifier bridge and a filter circuit becomes a stable DC voltage that is boosted to $9 \mathrm{~V}$ by boost module 1 . The super capacitor group is steadily charged by $9 \mathrm{~V}$ 's voltage through the MOSFET switch A and the equalizing circuit. When the voltage at both ends of the super capacitor group is detected to reach $9 \mathrm{~V}$, the DSP controller outputs two pulse width modulation signals. One output, pulse width modulation 2, is high and it drives the MOSFET switch B to open. The other output, pulse width modulation 1, is low and it drives the MOSFET switch A to close. At this moment, the super capacitor group is in discharge status and the output voltage of the super capacitor grope is boosted to $14.4 \mathrm{~V}$ by boost module 2 . The storage battery is steadily charged by $14.4 \mathrm{~V}$ 's voltage through voltage stabilizing circuit. In order to prevent over-discharge of the super capacitor group, the minimum discharge voltage of the super capacitor group is set at $4 \mathrm{~V}$, that is, when the voltage sensor detects that the voltage between the two ends of the super capacitor group reaches $4 \mathrm{~V}$, the mode is converted, and the whole system performs cyclic charge and discharge. 


\section{Nonlinear Modeling of the Semi-Active Energy Regenerative Suspension with an EHA}

\subsection{Dynamic Model of a Two Degrees of Freedom Suspension System for a Quarter-Vehicle}

In this paper, a quarter-car model with two degrees of freedom was exactly established [17], which is shown in Figure 3.

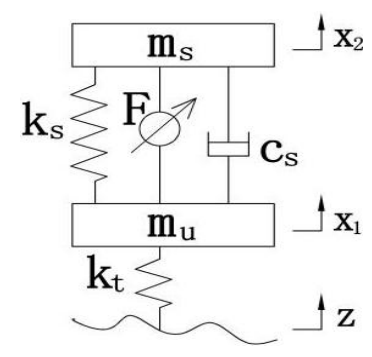

Figure 3. The schematic diagram of the quarter-car model.

According to Figure 3, the equations of motion are obtained by using Newton's laws of motion:

$$
\left\{\begin{array}{l}
m_{s} \ddot{x}_{2}+k_{s}\left(x_{2}-x_{1}\right)+c_{s}\left(\dot{x}_{2}-\dot{x}_{1}\right)=F \\
m_{u} \ddot{x}_{1}-k_{s}\left(x_{2}-x_{1}\right)-c_{s}\left(\dot{x}_{2}-\dot{x}_{1}\right)+k_{t}\left(x_{1}-z\right)=-F .
\end{array}\right.
$$

The state vector and output vector are selected as follows:

$$
\begin{gathered}
\boldsymbol{X}=\left[\begin{array}{llll}
x_{2}-x_{1} & \dot{x}_{2} & x_{1}-z & \dot{x}_{1}
\end{array}\right]^{\mathrm{T}} \\
\boldsymbol{Y}=\left[\begin{array}{llll}
\ddot{x}_{2} & x_{2}-x_{1} & k_{t}\left(x_{1}-z\right) & \dot{x}_{1}
\end{array}\right]^{\mathrm{T}}
\end{gathered}
$$

where $\ddot{x}_{2}$ is the sprung mass acceleration, $x_{2}-x_{1}$ is the suspension deflection response, $k_{t}\left(x_{1}-x_{0}\right)$ is the tire load response, and $\dot{z}(t)$ is the speed excitation of the road input. In this way, the state equation of the suspension can be obtained as follows:

$$
\left\{\begin{array}{l}
\dot{X}=A X+B U \\
Y=C X+D U
\end{array}\right.
$$

where $A$ is the state matrix, $B$ is the input matrix, $C$ is the output matrix, and $D$ is the transfer matrix. When the control input force $F$ is 0 , it becomes a passive suspension.

$$
\begin{gathered}
\boldsymbol{A}=\left[\begin{array}{cccc}
0 & 1 & 0 & -1 \\
-\frac{k_{s}}{m_{s}} & -\frac{c_{s}}{m_{s}} & 0 & \frac{c_{s}}{m_{s}} \\
0 & 0 & 0 & 1 \\
\frac{k_{s}}{m_{u}} & \frac{c_{s}}{m_{u}} & -\frac{k_{t}}{m_{u}} & -\frac{c_{s}}{m_{u}}
\end{array}\right] \boldsymbol{B}=\left[\begin{array}{cc}
0 & 0 \\
0 & \frac{1}{m_{s}} \\
-1 & 0 \\
0 & -\frac{1}{m_{u}}
\end{array}\right] \\
\boldsymbol{C}=\left[\begin{array}{cccc}
-\frac{k_{s}}{m_{s}} & -\frac{c_{s}}{m_{s}} & 0 & \frac{c_{s}}{m_{s}} \\
1 & 0 & 0 & 0 \\
0 & 0 & k_{t} & 0 \\
0 & 0 & 0 & 1
\end{array}\right] \boldsymbol{D}=\left[\begin{array}{cc}
0 & \frac{1}{m_{s}} \\
0 & 0 \\
0 & 0 \\
0 & 0
\end{array}\right] \boldsymbol{U}=\left(\begin{array}{c}
\dot{z} \\
F
\end{array}\right)
\end{gathered}
$$

The road surface input model adopts filtered white noise [18] as follows:

$$
\dot{z}(t)=-2 \pi f_{0} z(t)+2 \pi \sqrt{G_{0} u} \omega(t)
$$

where $z$ is the input displacement of the road, $G_{0}$ is the road irregularities coefficient, $f_{0}$ is the lower cutoff frequency, $u_{0}$ is the vehicle speed, and $\omega(t)$ is unit white noise. 


\subsection{Parameter Identification of Nonlinear Model}

In order to provide experimental sample data for the model parameter identification, a physical prototype of an EHA, which is mainly composed of a hydraulic cylinder, a hydraulic motor, and a brushless DC motor, is developed, and according to the national standards of QC/T545-1999 "Test method for automobile telescopic shock absorber", a force characteristics test of an EHA semi-active suspension system is carried out on an ES-6-230 numerical control hydraulic vibration table as shown in Figure 4. In the experiment, the vibration table input excitation uses sinusoidal input at the frequency of $1 \mathrm{~Hz}$ and the amplitude of $30 \mathrm{~mm}$. An LTR-1 pull pressure sensor is used to collect the suspension's output force signal in the passive state, and the change of the suspension's output force with time is shown in Figure 5. In order to eliminate the influence of initial conditions on the test results, the sampling time starts from $5 \mathrm{~s}$.

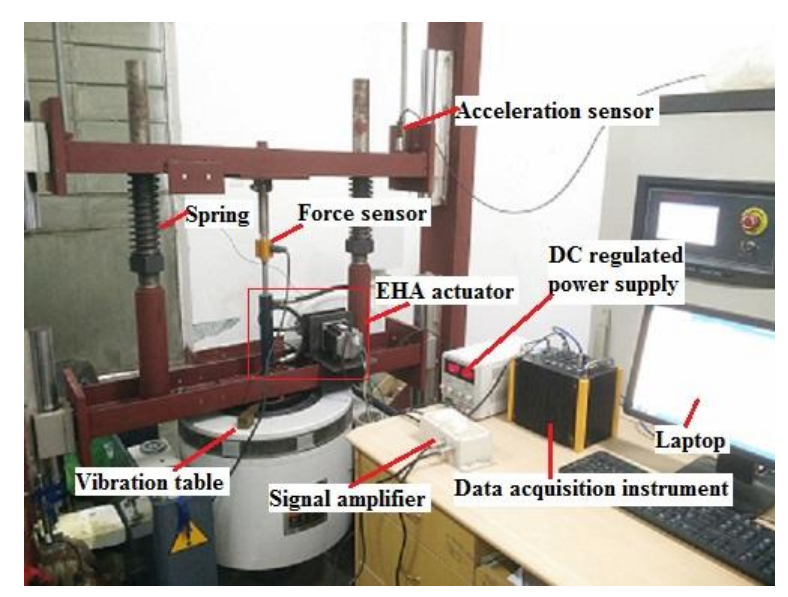

Figure 4. Force characteristic test of the EHA semi-active suspension system.

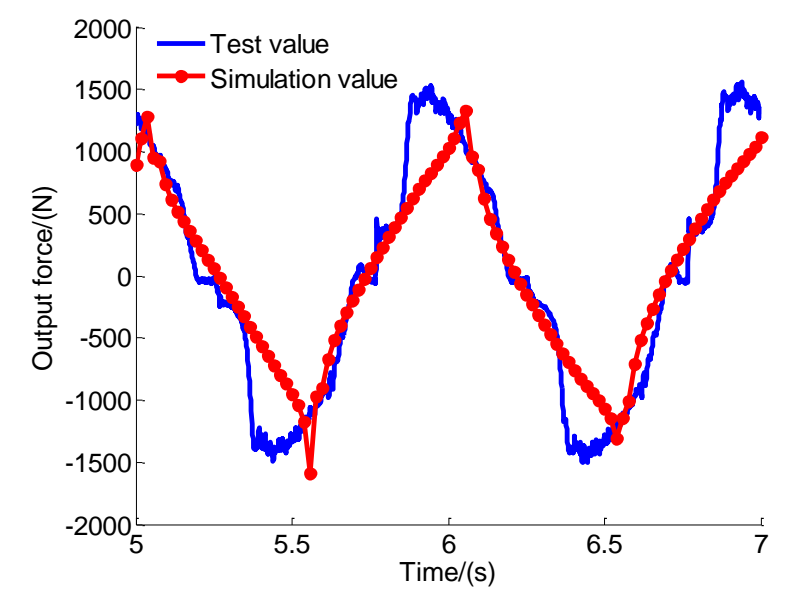

Figure 5. Comparison of simulation and experimental results of output force.

The parameters of the nonlinear model are continuously approximated by the least squares method based on the experimental data, and the objective function of the algorithm is expressed as:

$$
\left\{k_{s}, c_{s}\right\}=\underset{k_{s}, c_{s}}{\operatorname{argmin}}\left\|F_{\mathcal{c}}\left(k_{s}, c_{s}\right)-F_{e}\right\|_{2}^{2}
$$

where $k_{s}$ is the equivalent spring stiffness, $c_{s}$ is the inherent damping coefficient of the system, $F_{c}$ is the theoretical model value, and $F_{e}$ is the actual test value. 
Through the identification of the parameters, the equivalent spring stiffness $k_{s}$ of the EHA semi-active suspension system is $13,000 \mathrm{~N} / \mathrm{m}$, and the inherent damping coefficient $c_{S}$ is $500 \mathrm{~N} \cdot \mathrm{m} / \mathrm{s}$. The above parameters are introduced into the two degrees of freedom suspension dynamics model of the quarter-vehicle, and the simulation results of the EHA actuator output force are compared with the test results, as shown in Figure 5.

Figure 5 shows that the output force of the EHA actuator obtained by parameter identification is in good agreement with the actual output force, which indicates that the established dynamic model of the two degrees of freedom suspension system for a quarter-vehicle is accurate, and the adopted method of model parameter identification is feasible and effective.

\subsection{Mathematical Model of the Semi-Active Energy Regenerative Suspension with an EHA}

When the mathematical model of the EHA actuator is established, the friction between the piston, the cylinder wall, and the internal leakage of the system is ignored. Thus, the damping force generated by the hydraulic cylinder can be expressed as:

$$
F=\Delta P \cdot A
$$

where $\Delta P$ is the pressure drop between the upper and lower surface of the piston in the hydraulic cylinder, and $A$ is the effective piston area.

We take into account the hydraulic pipeline's pressure loss, which includes the pressure loss along the path and the local pressure loss [19].

The pressure loss along the path of the hydraulic pipeline in the system is expressed as:

$$
\Delta P_{\lambda}=\lambda \frac{l}{d}\left(\frac{\rho v^{2}}{2}\right)
$$

where $\lambda$ is the along resistance coefficient, $l$ is the length of the hydraulic pipe, $d$ is the diameter of the hydraulic pipe, $\rho$ is the hydraulic oil's density, and $v$ is the oil flow rate in the pipeline.

The local pressure loss of the hydraulic pipeline in the system is expressed as:

$$
\Delta P_{\zeta}=\zeta \frac{\rho v^{2}}{2}
$$

where $\zeta$ is the local resistance coefficient.

It can be seen that the total pressure loss of the hydraulic pipeline in the system can be obtained as follows:

$$
\Delta P_{g}=\Delta P_{\lambda}+\Delta P_{\zeta}=\left(\lambda \frac{l}{d}+\zeta\right) \frac{\rho v^{2}}{2}
$$

according to the continuity equation of liquid flow, which is given by:

$$
Q_{d}=A \cdot v_{g}=\frac{\pi d^{2}}{4} v
$$

where $Q_{d}$ is the flow of the hydraulic motor, and $v_{g}$ is the speed of the piston rod.

According to the working principle of the semi-active energy regenerative suspension with an EHA, it can be seen that under the action of body vibration the oil in the hydraulic cylinder enters the hydraulic motor and drives the hydraulic motor to work, and the output torque of the hydraulic motor drives the generator to generate electricity. At the same time, the angular velocity and output torque of the hydraulic motor meet the following relationships:

$$
\begin{aligned}
& \omega_{0}=\frac{2 \pi Q_{d}}{q} \eta_{v} \\
& T_{d}=\frac{\Delta p_{d} q}{2 \pi} \eta_{m}
\end{aligned}
$$


where $q$ is the hydraulic motor displacement, $\eta_{v}$ is the volumetric efficiency of the hydraulic motor, $\Delta p_{d}$ is the pressure drop between the inlet and the outlet of the hydraulic motor, and $\eta_{m}$ is the mechanical efficiency of the hydraulic motor.

The generator converts the mechanical energy of the hydraulic motor into electrical energy, so the input torque of the generator is equal to the output torque of the hydraulic motor. Therefore, the output voltage $U$ and the output torque $T_{g}$ of the generator meet the following relationships:

$$
\begin{gathered}
U=E-I(r+R)=I R_{0} \\
T_{g}=J \ddot{\theta}+k_{t} I
\end{gathered}
$$

where $E$ is the induced electromotive force, $I$ is the loop current, $r$ is the internal resistance of the generator, $R$ is the external load resistance of the generator, $R_{0}$ is the equivalent resistance of the energy regenerative circuit, $J$ is the moment of inertia, $\ddot{\theta}$ is the angular acceleration of the generator, and $k_{t}$ is the torque constant of the generator.

Among them, the induction electromotive force is expressed as:

$$
E=k_{e} n=\frac{30}{\pi} k_{e} \omega
$$

where $k_{e}$ is the back electromotive force constant of the generator, $n$ is the generator rotor speed, and $\omega$ is the generator rotor angular velocity.

The hydraulic motor is directly connected with the generator through the coupling, so their angular velocity and torque are equal, which is expressed as $\omega_{0}=\omega$ and $T_{d}=T_{g}$. If the moment of inertia of the motor is ignored, the pressure drop between the inlet and the outlet of the hydraulic motor can be obtained from Equations (9)-(14) as follows:

$$
\Delta P_{d}=\frac{120 \pi k_{e} k_{t} \eta_{v} A v_{g}}{q^{2} \eta_{m}\left(r+R+R_{0}\right)} .
$$

The pressure balance equation of the whole hydraulic circuit be expressed as:

$$
\Delta P=\Delta P_{d}+\Delta P_{g}=\frac{120 \pi k_{e} k_{t} \eta_{v} A v_{g}}{q^{2} \eta_{m}\left(r+R+R_{0}\right)}+\left(\lambda \frac{l}{d}+\zeta\right) \frac{8 \rho A^{2} v_{g}^{2}}{\pi^{2} d^{4}}
$$

The damping force of the EHA actuator can be obtained by taking Equation (16) into Equation (14) as follows:

$$
F=\frac{120 \pi k_{e} k_{t} \eta_{v} A^{2} v_{g}}{q^{2} \eta_{m}\left(r+R+R_{0}\right)}+\left(\lambda \frac{l}{d}+\zeta\right) \frac{8 \rho A^{3} v_{g}^{2}}{\pi^{2} d^{4}} .
$$

Therefore, the equivalent damping coefficient of the EHA actuator can be expressed as:

$$
c_{e q}=\frac{120 \pi k_{e} k_{t} \eta_{v} A^{2}}{q^{2} \eta_{m}\left(r+R+R_{0}\right)}+\left(\lambda \frac{l}{d}+\zeta\right) \frac{8 \rho A^{3} v_{g}}{\pi^{2} d^{4}} .
$$

The loop current can be obtained from Equations (11), (13), and (15) as follows:

$$
I=\frac{60 k_{e} \eta_{v} A v_{g}}{q\left(r+R+R_{0}\right)}+\left(\lambda \frac{l}{d}+\zeta\right) \frac{4 \rho q \eta_{m} A^{2} v_{g}^{2}}{\pi^{3} d^{4} k_{t}} .
$$

In summary, the instantaneous energy regenerative power can be expressed as:

$$
P_{\text {reg }}=I^{2} R_{0}=\left[\frac{60 k_{e} \eta_{v} A v_{g}}{q\left(r+R+R_{0}\right)}+\left(\lambda \frac{l}{d}+\zeta\right) \frac{4 \rho q \eta_{m} A^{2} v_{g}^{2}}{\pi^{3} d^{4} k_{t}}\right]^{2} R_{0}
$$

In the traditional passive suspension damper, the dissipated power in the form of heat energy is calculated as:

$$
P_{c o n}=c_{s}\left(\dot{x}_{2}-\dot{x}_{1}\right)^{2}
$$


where $c_{s}$ is the inherent damping coefficient, and $\left(\dot{x}_{2}-\dot{x}_{1}\right)$ is the shock absorber velocity.

The energy regenerative efficiency of a semi-active suspension is the ratio between the feedback energy and the dissipated energy of the passive suspension. Therefore, the energy regenerative efficiency $\eta$ is expressed as:

$$
\eta=\frac{\int_{0}^{T} P_{r e g} \mathrm{~d} t}{\int_{0}^{T}\left[c_{s}\left(\dot{x}_{2}-\dot{x}_{1}\right)^{2}\right] \mathrm{d} t} .
$$

\section{The Control Strategy of the Semi-Active Energy Regenerative Suspension with an EHA}

The selection principle of switching the control critical point to control the force of the semi-active energy regenerative suspension with an EHA is expressed as follows:

When $\dot{x}_{2} \cdot\left(\dot{x}_{2}-\dot{x}_{1}\right)>0$, the direction of the force applied to the spring mass by the actuator is opposite to the movement direction of the spring mass, but it has the same direction as the active control force, so it can change the external load resistance of the actuator to make the actual control output force be equal to the theoretical active control output force. Additionally, the theoretical active control output force is determined by the optimal control strategy.

The optimal control objective of the semi-active energy regenerative suspension with an EHA is to make the car obtain higher comfort and handling stability, and reflecting in the amount of actual control is to reduce the acceleration of the sprung mass and tire dynamic load as much as possible, limit the range of the suspension's dynamic deflection, and reduce the possibility of suspension impact block. At the same time, it should not consume too much energy [20]. Based on the above considerations, the performance index function of a semi-active suspension output regulator can be written as follows:

$$
J=\int_{0}^{\infty}\left[q_{1} \ddot{x}_{2}^{2}+q_{2}\left(x_{2}-x_{1}\right)^{2}+q_{3}\left(x_{1}-z\right)^{2}+r F^{2}\right] \mathrm{d} t
$$

where $q_{1}$ is the weighting coefficient of acceleration, $q_{2}$ is the weighting coefficient of the suspension's dynamic deflection, $q_{3}$ is the weighting coefficient of tire dynamic deformation, and $r$ is the weighting coefficient of energy consumption.

The above optimization index is expressed in matrix as:

$$
J=\int_{0}^{\infty}\left[Y^{T} q Y+F^{T} r F\right] \mathrm{d} t
$$

where $q$ is expressed as:

$$
q=\left[\begin{array}{ccc}
q_{1} & 0 & 0 \\
0 & q_{2} & 0 \\
0 & 0 & q_{3}
\end{array}\right]
$$

Generally, the output regulator problem is converted to a state regulator problem. Taking the output equation $Y=C X+D U$ into Equation (24), the quadratic performance index is given by:

$$
J=\int_{0}^{\infty}\left[X^{T} Q X+2 X^{T} N F+F^{T} R F\right] \mathrm{d} t
$$

where $Q$ are the positive semidefinite symmetric weighting matrices of the state variables, $N$ is the weighted matrix of two kinds of variables, and $R$ is the positive definite symmetric weighting matrix of the control variables. Among them, $Q=C^{T} q C, N=C^{T} q D, R=r+D^{T} q D$.

The optimal control force $F$, which serves for performance index $J$, to be minimized is existent and unique, and it can be expressed as:

$$
F=-K X=-\left(B^{T} P+N^{T}\right) X
$$

where $P$ is a symmetric positive definite solution of the Riccati matrix equation. It meets the following equation: 


$$
P \mathrm{~A}+\mathrm{A}^{\mathrm{T}} P-(P \mathrm{~B}+\mathrm{N}) R^{-1}\left(\mathrm{~B}^{\mathrm{T}} P+\mathrm{N}^{\mathrm{T}}\right)+Q=0 .
$$

The selection of the weighting factors in the performance index of the optimal control depends on practical experience. After repeated trials, the following options can be made: $q_{1}=1.2 \times 10^{5}$, $q_{2}=1.65 \times 10^{8}, q_{3}=9.5 \times 10^{9}, r=1$.

In order to obtain the feedback gain matrix $K$, using the linear quadratic regulator function provided by Matlab software, the basic format is expressed as:

$$
(K, S, E)=L Q R(A, B, Q, R, N)
$$

where $S$ is the solution of the Riccati equation, and $E$ is the system eigenvalue.

When $\dot{x}_{2} \cdot\left(\dot{x}_{2}-\dot{x}_{1}\right)<0$, the direction of the force applied to the spring mass by the actuator has the same direction as the movement direction of the spring mass, but it is opposite to that of the active control force. In order to minimize this difference, at this moment the damping force, which is theoretically required to output from the suspension, is zero, but the actual output force is the inherent viscous damping force of the system, and its true value is still determined by Formula (17). At the same time, the motor load resistance is zero.

\section{Parameter Optimization of the Semi-Active Energy Regenerative Suspension with an EHA}

\subsection{Parameter Sensitivity Analysis}

In order to coordinate the damping performance and energy regenerative performance of the semi-active energy regenerative suspension with an EHA and improve the energy regenerative performance under the condition of satisfying the requirement of damping performance, the influence of the EHA's parametric variation on the damping performance and energy regenerative performance of the suspension is analyzed. The EHA is mainly composed of a hydraulic cylinder, a hydraulic motor, and a brushless DC generator. According to the modeling process, it can be known that the main parameters of the EHA are: the effective area of the hydraulic cylinder piston $A$, the displacement of the hydraulic motor $q$, and the back electromotive force constant of the generator $k_{e}$. Based on the initial simulation parameters' value of the EHA and $20 \%$ of the initial simulation parameters' value of the EHA for the change interval, the influence of the three main parameters' variation of the EHA on the damping performance and energy regenerative performance of the semi-active energy regenerative suspension with the EHA is investigated. Among them, the damping performance takes spring mass acceleration and tire dynamic load as evaluation indicators, and energy regenerative performance takes energy regenerative power and energy regenerative efficiency as evaluation indicators. The influence of the main parameters of the EHA on the performance evaluation indicators is shown in Figures 6-8, respectively.

Figure 6 shows that, firstly, with the increase of the effective area of the hydraulic cylinder piston, the RMS values of the sprung mass acceleration and the dynamic load of the tire decrease gradually. Then, they reach the minimum at nearly 1.2 times the value of the original simulation parameters of the hydraulic cylinder piston's effective area, which makes riding comfort continuously improve and the damping performance achieve an optimal effect. Finally, with the increase of the effective area of the hydraulic cylinder piston, the RMS values of the sprung mass acceleration and the dynamic load of the tire increase gradually, which leads to deterioration of ride comfort and the damping performance. However, with the increase of the effective area of the hydraulic cylinder piston, the RMS values of the energy regenerative power and the energy regenerative efficiency increase gradually, that is to say, the energy regenerative performance improves continuously. However, when the RMS values of the energy regenerative power and the energy regenerative efficiency reach the maximum, which means that the energy regenerative efficiency is optimal, the RMS values of the sprung mass acceleration and the dynamic load of the tire reach the maximum too, which leads to damping performance deterioration without meeting the design requirements of the suspension. 


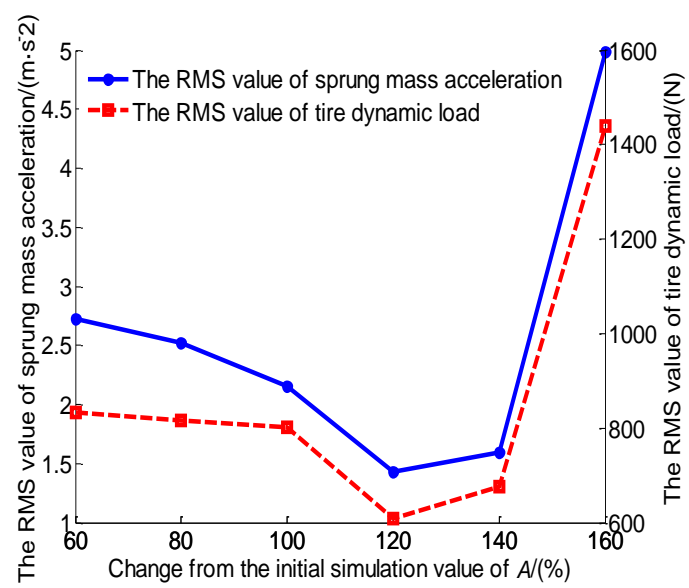

(a)

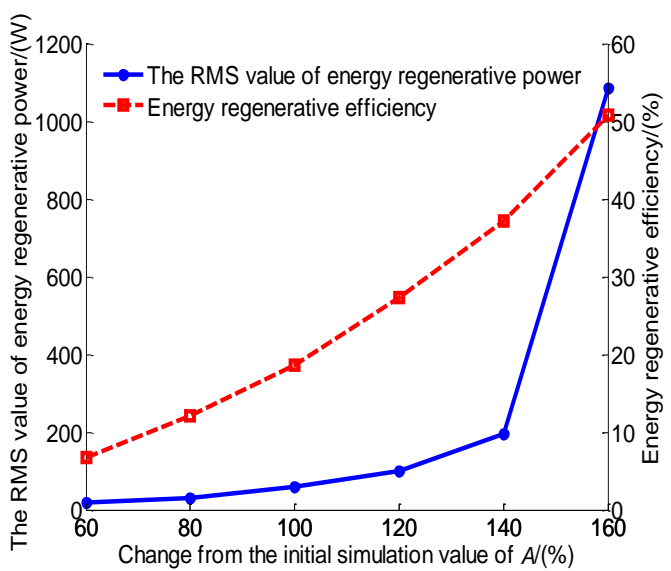

(b)

Figure 6. Influence of the effective area of the hydraulic cylinder piston on evaluation indicators:

(a) Influence of the effective area of the hydraulic cylinder piston on the damping performance;

(b) Influence of the effective area of the hydraulic cylinder piston on energy regenerative performance.

Figure 7 shows that, firstly, with the increase of the displacement of the hydraulic motor, the RMS values of the sprung mass acceleration and the dynamic load of the tire decrease gradually. Then, they reach the minimum at nearly 0.8 times the value of the original simulation parameters of the displacement of the hydraulic motor, which makes riding comfort continuously improve and the damping performance achieve an optimal effect. Finally, with the increase of the displacement of the hydraulic motor, the RMS values of the sprung mass acceleration and the dynamic load of the tire increase gradually, which leads to the deterioration of ride comfort and damping performance. However, with the increase of the displacement of the hydraulic motor, the RMS values of the energy regenerative power and the energy regenerative efficiency decrease gradually, that is to say, the energy regenerative performance deteriorates continuously. However, when the RMS values of the energy regenerative power and the energy regenerative efficiency reach the maximum, which means that the energy regenerative efficiency is optimal, the RMS values of the sprung mass acceleration and the dynamic load of the tire reach the maximum too, which leads to damping performance deterioration without meeting the design requirements of the suspension.

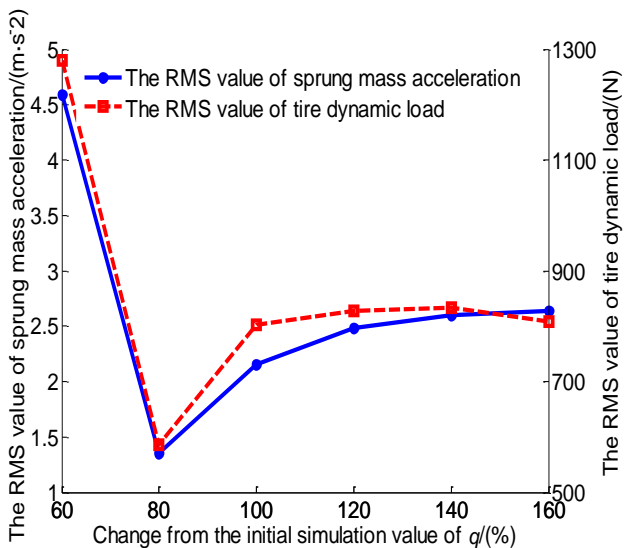

(a)

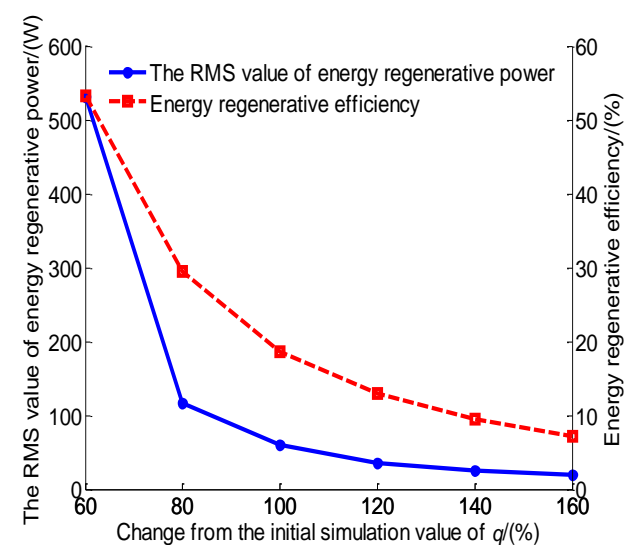

(b)

Figure 7. Influence of the displacement of the hydraulic motor on evaluation indicators: (a) Influence of the displacement of the hydraulic motor on the damping performance; (b) Influence of the displacement of the hydraulic motor on energy regenerative performance. 
Figure 8 simulation results show that, firstly, with the increase of the back electromotive force constant of the generator, the RMS values of the sprung mass acceleration and the dynamic load of the tire decrease gradually. Then, they reach the minimum at nearly 1.8 times the value of the original simulation parameters of the back electromotive force constant of the generator, which makes riding comfort continuously improve and the damping performance achieve an optimal effect. Finally, with the increase of the back electromotive force constant of the generator, the RMS values of the sprung mass acceleration and the dynamic load of the tire increase gradually, which leads to the deterioration of ride comfort and damping performance. However, with the increase of the back electromotive force constant of the generator, the RMS values of the energy regenerative power and the energy regenerative efficiency increase gradually, that is to say, energy regenerative performance improves continuously. However, when the RMS values of the energy regenerative power and the energy regenerative efficiency reach the maximum, which means that the energy regenerative efficiency is optimal, the RMS values of the sprung mass acceleration and the dynamic load of the tire reach the maximum too, which leads to damping performance deterioration without meeting the design requirements of the suspension.

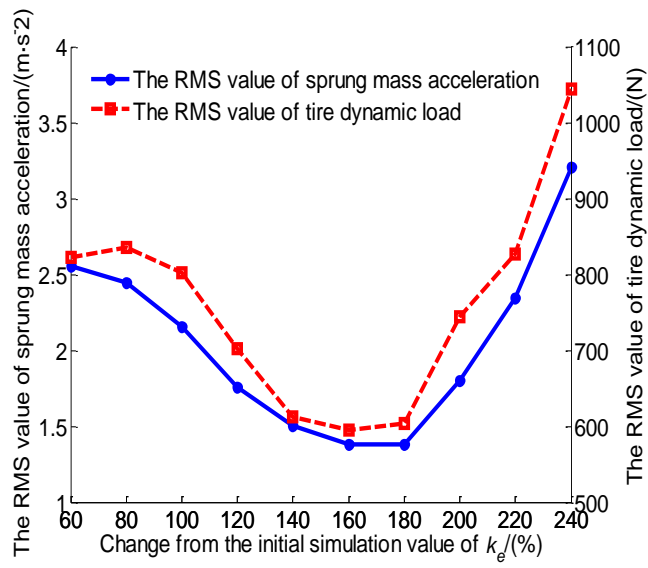

(a)

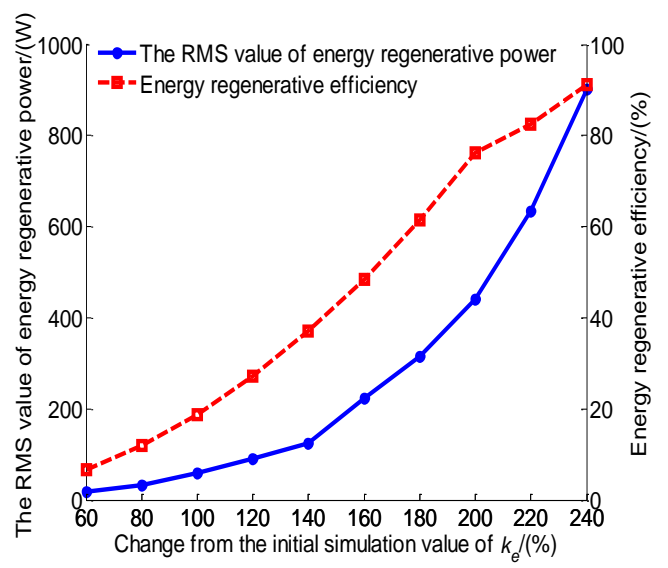

(b)

Figure 8. Influence of the back electromotive force constant of the generator on evaluation indicators: (a) Influence of the back electromotive force constant of the generator on the damping performance; (b) Influence of the back electromotive force constant of the generator on energy regenerative performance.

From the above study, it can be concluded that with the change of parameters of the EHA, the damping performance and energy regenerative performance cannot achieve an optimal effect at the same time, and there is mutual restriction between them. In order to balance the damping performance and energy regenerative performance, the three main parameters of the EHA, including the effective area of the hydraulic cylinder piston $A$, the displacement of the hydraulic motor $q$, and the back electromotive force constant of the generator $k_{e}$, should be coordinated and optimized.

\subsection{Optimization Objectives and Constraints}

In order to improve the energy regenerative performance under the requirement for meeting a certain damping performance, the genetic algorithm is used to optimize the parameters of the EHA, which can reduce the risk of being trapped in a locally optimal solution and make the optimization results more accurate [21,22].

The parameter optimization of the semi-active energy regenerative suspension with an EHA is a nonlinear optimization problem of a single target and multiple variables. Taking energy regenerative power as the optimization goal, taking a certain damping performance as the constraint condition, 
and taking the effective area of the hydraulic cylinder piston $A$, the displacement of the hydraulic motor $q$, and the back electromotive force constant of the generator $k_{e}$ as optimization variables, the genetic algorithm optimization toolbox from Matlab is used to optimize the parameters.

The objective function is the RMS value $\sigma_{\text {Preg }}$ of the energy regenerative power of the semi-active energy regenerative suspension with an EHA, and $\sigma_{\text {Preg }}$ is given by:

$$
\sigma_{P_{\text {reg }}}=\sqrt{\frac{\sum_{i=1}^{N} P_{\text {reg }}{ }^{2}}{N}} .
$$

In the optimization toolbox, the objective function is required to be minimized. In this paper, however, the RMS value of the energy regenerative power is required to be maximized, so the method to minimize the negative value of the objective function is feasible.

The constraint condition in this paper is to meet a certain damping performance. According to automobile theory and other related literatures, the damping of vehicle suspension belongs to small damping, and the damping ratio $\xi$ meets the condition $0.2 \leq \xi \leq 0.4$. Additionally, when the RMS value of the wheel dynamic load $\sigma_{F_{d}}$ does not exceed $1 / 3$ of the static load value, the probability of a wheel jumping off the ground is less than $0.15 \%$, which can ensure the comfort and safety of the suspension [23]. Meanwhile, the optimization toolbox requires that the constraint conditions are not positive. Thus, the constraint conditions meet:

$$
\left\{\begin{array}{c}
0.2-\frac{c_{s}+c_{e q}(X)}{2 \sqrt{k_{s} \cdot m_{s}}} \leq 0 \\
\frac{c_{s}+c_{e q}(X)}{2 \sqrt{k_{s} \cdot m_{s}}}-0.4 \leq 0 \\
\sigma_{F_{d}}(X)-\frac{1}{3} G \leq 0
\end{array}\right.
$$

where $X=\left\{x_{1}, x_{2}, x_{3}\right\}=\left\{A, q, k_{e}\right\}$ is the optimal parameter vector.

\subsection{Optimization Result Analysis}

The above parameters are brought into the genetic algorithm optimization toolbox, and the optimization results of the semi-active energy regenerative suspension with an EHA are shown in Table 1.

Table 1. Optimal parameters.

\begin{tabular}{ccc}
\hline Optimal Parameters & Symbol & Value \\
\hline The effective area of the hydraulic cylinder piston & $A$ & $7.66 \times 10^{-4} \mathrm{~m}^{2}$ \\
The displacement of the hydraulic motor & $q$ & $4.25 \mathrm{~mL} / \mathrm{r}$ \\
The back electromotive force constant of the generator & $k_{e}$ & $8.28 \times 10^{-3} \mathrm{~V} \cdot \mathrm{min} / \mathrm{r}$ \\
\hline
\end{tabular}

In order to verify the effect of the optimized EHA's parameters on the performances of the semi-active energy regenerative suspension with the EHA, the performance indexes of the suspension before and after optimization are simulated and compared assuming that the vehicle is traveling at a speed of $20 \mathrm{~m} / \mathrm{s}$ on a C-grade road and the simulation time is $10 \mathrm{~s}$ [24]. Additionally, the simulation results are shown in Table 2 and Figures 9-11.

Table 2 shows that after optimizing the parameters, the RMS value of the sprung mass acceleration is reduced by $18.60 \%$, which indicates that the ride comfort of the vehicle has been improved to a certain extent, and the RMS value of the tire dynamic load is decreased by $12.61 \%$, which shows that the vehicle's driving safety has been enhanced; therefore, the vehicle's damping performance is improved. After optimizing the parameters, the RMS value of the energy regenerative power increases by $49.67 \%$, 
and the energy regenerative efficiency increases from $18.68 \%$ to $27.26 \%$, which demonstrates that the vehicle's energy regenerative performance was improved significantly.

Table 2. Simulation results.

\begin{tabular}{ccccc}
\hline \multirow{2}{*}{ Performance Index } & \multirow{2}{*}{ Symbol } & \multicolumn{2}{c}{ Value } & \multirow{2}{*}{ Control Effect/\% } \\
\cline { 3 - 4 } & & Before Optimization & After Optimization & \\
\hline Sprung mass acceleration & $\sigma_{a}$ & $2.1585 \mathrm{~m} / \mathrm{s}^{2}$ & $1.7571 \mathrm{~m} / \mathrm{s}^{2}$ & -18.60 \\
Tire dynamic load & $\sigma_{F d}$ & $802.7 \mathrm{~N}$ & $701.5 \mathrm{~N}$ & -12.61 \\
Energy regenerative power & $\sigma_{P r e g}$ & $59.82 \mathrm{~W}$ & $89.53 \mathrm{~W}$ & 49.67 \\
Energy regenerative efficiency & $\eta$ & $18.68 \%$ & $27.26 \%$ & 45.93 \\
\hline
\end{tabular}

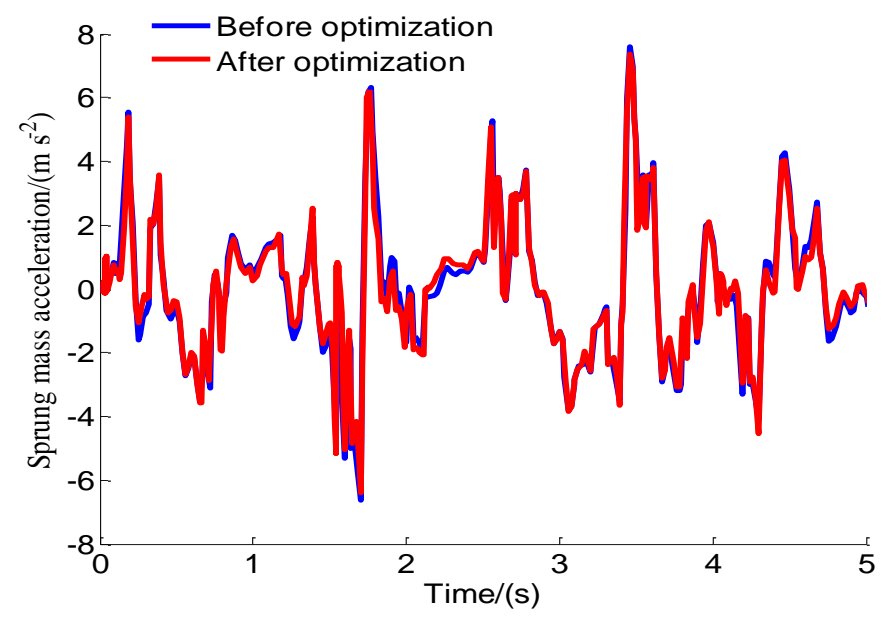

Figure 9. Spring mass acceleration response curve.

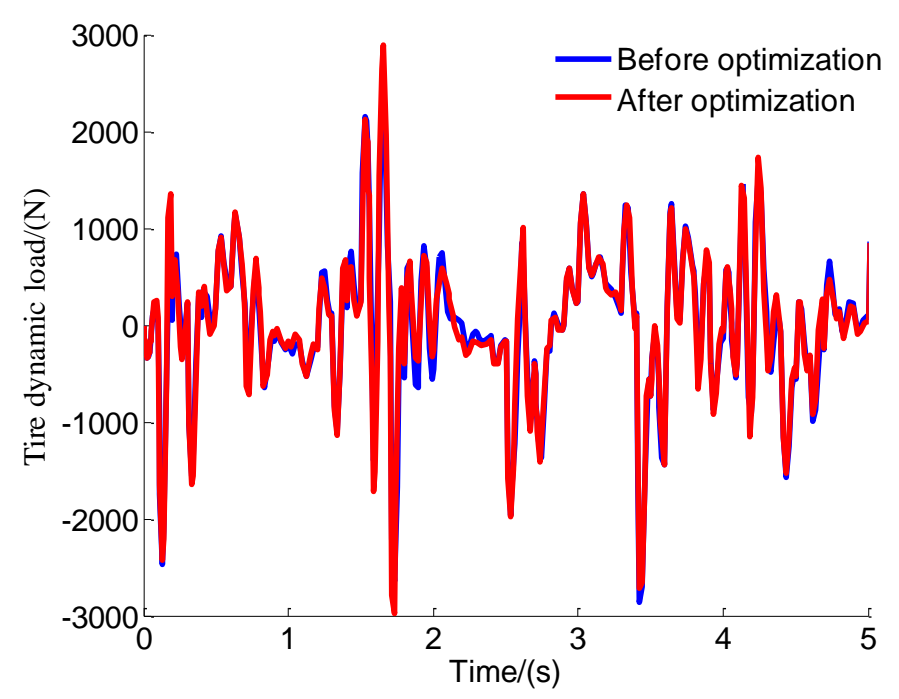

Figure 10. Tire dynamic load response curve. 


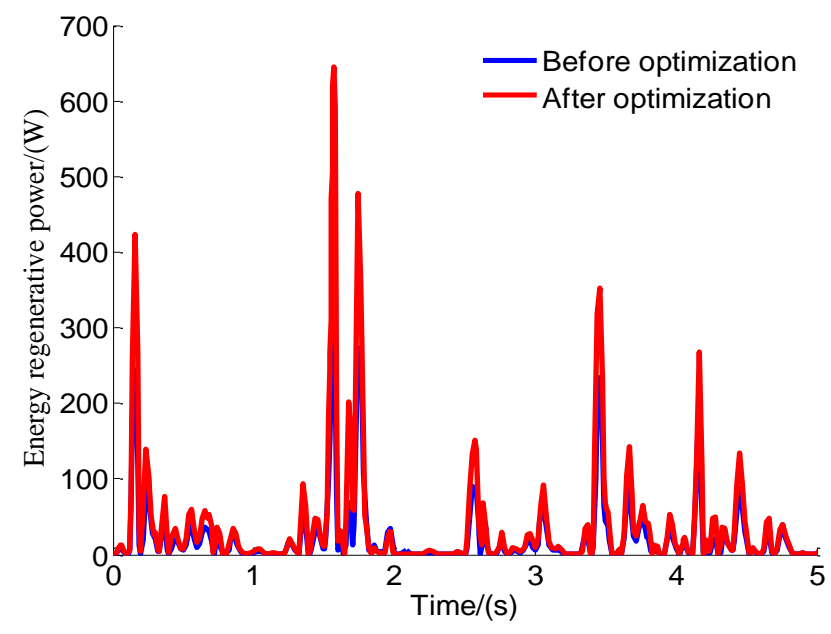

Figure 11. Energy regenerative power response curve.

\subsection{Test and Analysis}

In order to further verify the optimization results, the EHA prototype is redeveloped based on the optimization results, and the bench test is carried out as shown in Figure 12. In addition, the EHA actuator in Figure 4 is the original EHA actuator before optimization, and the EHA actuator in Figure 12 is the optimized one. Due to the limitation of test conditions, the test is conducted only for the spring mass acceleration and the energy regenerative power of the semi-active suspension with an EHA. In the test, the DH186 acceleration sensor produced by Donghua testing company is used to collect the spring mass acceleration signal, and the rectifier is used to rectify the three-phase alternating current generated by the DC brushless motor. At the same time, the Donghua DH5902 data acquisition system is used to collect the feed voltage signal. Additionally, according to the relationship between power, voltage, and the internal resistance of the motor, the instantaneous energy regenerative power can be obtained. Under the condition that the sinusoidal is input at the frequency of $2 \mathrm{~Hz}$, the amplitude of $30 \mathrm{~mm}$, and the sampling time of $10 \mathrm{~s}$, the test results of the spring mass acceleration response and energy regenerative power response of the semi-active suspension with an EHA before and after optimization are shown in Figures 13 and 14, respectively.

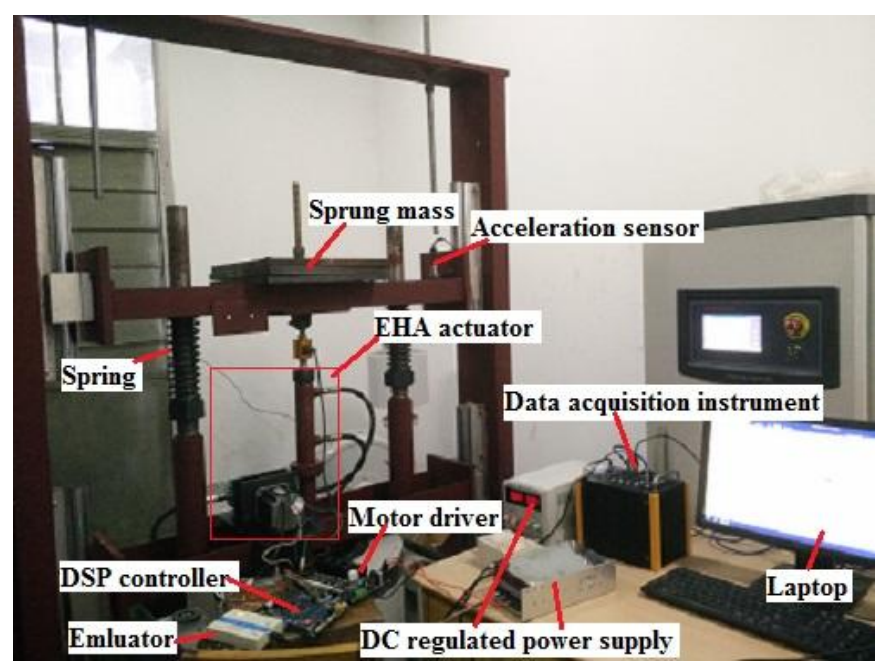

Figure 12. Bench test of EHA semi-active suspension system. 


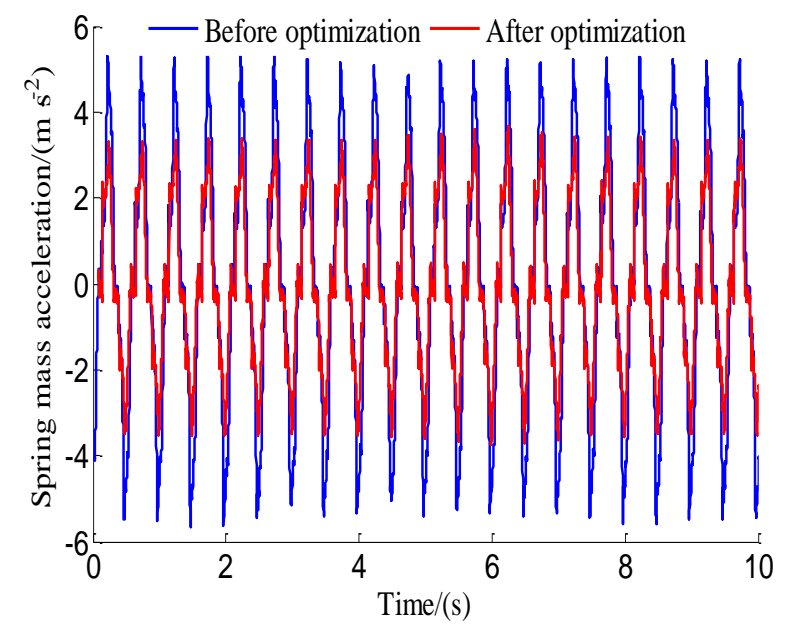

Figure 13. Test chart of spring mass acceleration response.

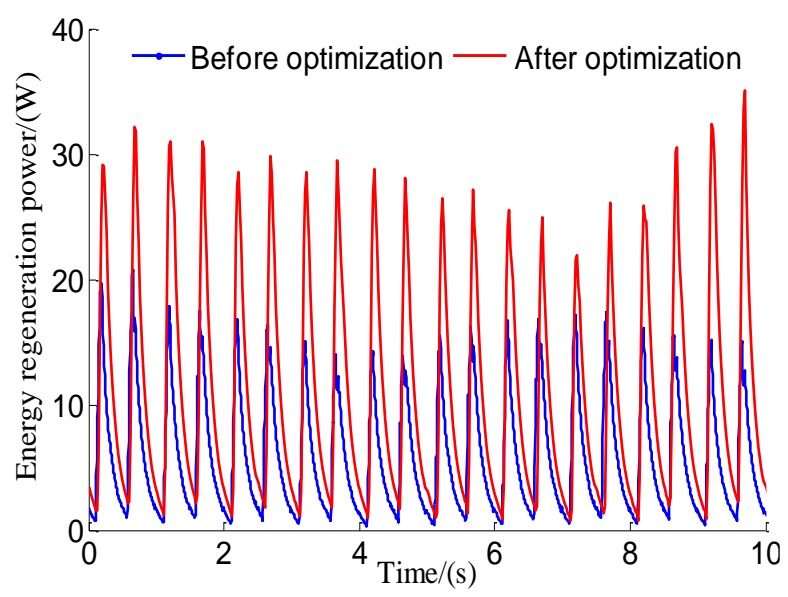

Figure 14. Test chart of energy regenerative power response.

\section{Conclusions}

In this paper, a structure of a semi-active energy regenerative suspension with an EHA is proposed. Following the proposed concept, a specific energy regenerative scheme and a composite energy recovery device are designed. On the basis of the system's structure, the physical prototypes are trial-manufactured, and a mechanical properties test of the EHA semi-active suspension system is carried out. Based on the experimental data, the parameter identification for the system model is conducted via a recursive least squares algorithm, which determines the equivalent spring stiffness $k_{s}$ and inherent damping coefficient of the system $c_{s}$. Moreover, the nonlinear model of the semi-active energy regenerative suspension with an EHA is built.

On the basis of the nonlinear model, the LQG control strategy of the semi-active energy regenerative suspension with an EHA is designed. Then, a complete simulation model of the semi-active energy regenerative suspension with an EHA is established in Simulink.

In order to coordinate the damping performance and energy regenerative performance of the semi-active energy regenerative suspension with an EHA, the influences of the main parameters of the EHA on the damping performance and energy regenerative performance of the suspension are analyzed. Finally, the main parameters of the EHA are optimized using the genetic algorithm.

In order to further verify the optimization results, the EHA prototype is redeveloped according to the optimization results, and the bench test is carried out. The test results show that when the sinusoidal is input at the frequency of $2 \mathrm{~Hz}$ and the amplitude of $30 \mathrm{~mm}$, the spring mass acceleration 
RMS value of the optimized semi-active energy regenerative suspension with an EHA is reduced by $22.23 \%$, and the energy regenerative power RMS value is increased by $40.51 \%$, which means that under meeting the requirements of vehicle ride comfort and driving safety, the energy regenerative performance is improved significantly.

Acknowledgments: The authors gratefully acknowledge the support of the National Natural Science Foundation of China (51775426, 51275403), the Service Local Special Program Support Project of Shaanxi Provincial Education Department (17JF017), and the Xi'an Science and Technology Project Funding Project (2017079CG/RC042-XAKD007). The authors would like to thank the anonymous reviewers for their valuable comments that significantly improved the quality of this paper.

Author Contributions: F.K. and J.D. conceived and designed the experiments; D.L. and J.X. performed the experiments; J.D. and Z.W. analyzed the data; J.D. wrote the paper.

Conflicts of Interest: The authors declare no conflict of interests.

\section{References}

1. Tseng, H.E.; Hrovat, D. State of the art survey: Active and semi-active suspension control. Veh. Syst. Dyn. 2015, 53, 1034-1062. [CrossRef]

2. Chen, S.A.; Li, X.; Zhao, L.J. Development of a control method for an electromagnetic semi-active suspension reclaiming energy with varying charge voltage in steps. Int. J. Automot. Technol. 2015, 16, 765-773. [CrossRef]

3. Wu, L.N.; Shi, M.M.; Wang, R.C. Parameter optimization of hybrid suspension with linear motor based on PSO. J. Chongqing Univ. Technol. (Nat. Sci.) 2016, 30, 12-17.

4. Babak, E. Development of Hybrid Electromagnetic Dampers for Vehicle Suspension Systems; University of Waterloo: Waterloo, ON, Canada, 2009.

5. Okada, Y.; Ozawa, K. Energy Regenerative and Active Control of Electro-Dynamic Vibration Damper. Trans. Jpn. Soc. Mech. Eng. C 2005, 70, 233-242.

6. Zhang, H.; Guo, X.X.; Hu, S.B.; Fang, Z.G.; Xu, L. Simulation analysis on hydraulic-electrical energy regenerative semi-active suspension control characteristic and energy recovery validation test. Trans. Chin. Soc. Agric. Eng. (Trans. CSAE) 2017, 33, 64-71.

7. Efatpenah, F.; Beno, J.H.; Weeks, D.A. Energy requirements of a passive and an electromechanical active suspension system. Veh. Syst. Dyn. 2000, 34, 437-458. [CrossRef]

8. Goldner, R.; Zerigian, P.; Hull, J. A Preliminary Study of Energy Recovery in Vehicles by Using Regenerative Magnetic Shock Absorbers; SAE Paper; SAE International: Warrendale, PA, USA, 2001; pp. 2001-2011.

9. Zuo, L.; Nayfeh, S.A. Structured H2 optimizatioensions based on multi-wheel models. Veh. Syst. Dyn. 2003, 40, 351-371. [CrossRef]

10. Anubi, O.M.; Clemen, L. Energy-regenerative model predictive control. J. Frankl. Inst. 2015, 352, 2152-2170. [CrossRef]

11. Clemen, L.; Margolis, D. Modeling and control of a quarter car electrodynamic air-suspension. In Proceedings of the International Conference on Bond Graph Modeling, Monterey, CA, USA, 6-10 July 2014; pp. 27-33.

12. Zuo, L.; Scully, B.; Shestani, J.; Zhou, Y. Design and characterization of an electromagnetic energy harvester for vehicle suspensions. Smart Mater. Struct. 2010, 19, 145-153. [CrossRef]

13. Zuo, L.; Tang, X.D. Large-scale vibration energy harvesting. J. Intell. Mater. Syst. Struct. 2013, 24, 1405-1430. [CrossRef]

14. Huang, K.; Zhang, Y.C.; Yu, F. Coordinate optimization for synthetical performance of electrical energy-regenerative active suspension. J. Shanghai Jiao Tong Univ. 2009, 43, 226-230.

15. Wang, Q.N.; Liu, S.S.; Wang, W.H. Structure design and parameter matching of ball-screw regenerative damper. J. Jilin Univ. Eng. Technol. 2012, 42, 1100-1106.

16. Kou, F.R. Design and energy regenerative study on semi-active suspension with electro-hydrostatic actuator. Trans. Chin. Soc. Agric. Mach. 2016, 28, 352-359.

17. Martins, I.; Estevez, J.; Marques, G. Permanent-magnets linear actuators applicability in automobile active suspensions. IEEE Trans. Veh. Technol. 2006, 55, 86-94. [CrossRef]

18. Kou, F.R.; Fan, Y.Q.; Zhang, C.W.; Du, J.F.; Wang, Z. Time delay compensation control of semi-active suspension with vehicle electro-hydrostatic actuator. China Mech. Eng. 2016, 27, 2111-2117. 
19. Sun, X.Q.; Chen, L.; Wang, S.H. Nonlinear modeling and parameter optimization of two-stage seriesconnected ISD suspension. Trans. Chin. Soc. Agric. Mach. 2014, 45, 7-13.

20. Kawamoto, Y.; Suda, Y.; Inoue, H. Electro-mechanical suspension system considering energy consumption and vehicle manoeuvre. Veh. Syst. Dyn. 2008, 46, 1053-1063. [CrossRef]

21. Kou, F.R.; Du, J.F.; Zhang, C.W.; Wang, Y.J.; Wang, Y.Z. Multi-mode switching control of self-powered active suspension with electro-hydrostatic actuator. Mech. Sci. Technol. Aerosp. Eng. 2016, 35, 1937-1943.

22. Li, C.; Zhu, R.R.; Liang, M. Integration of shock absorption and energy harvesting using a hydraulic rectifier. J. Sound Vib. 2014, 33, 3904-3916. [CrossRef]

23. Zhao, L.F.; Hu, J.F.; Zhang, R.Y. Integrated optimization design of cab suspension and suspension parameters for heavy tractor. China Mech. Eng. 2016, 27, 791-808.

24. Zhan, M. Dynamic Simulation and Performance Study of Hydraulic Electromagnetic Regenerative Shock Absorber; Jilin University: Changchun, China, 2015.

(C) 2018 by the authors. Licensee MDPI, Basel, Switzerland. This article is an open access article distributed under the terms and conditions of the Creative Commons Attribution (CC BY) license (http://creativecommons.org/licenses/by/4.0/). 